\title{
GLAD!
}

Revue sur le langage, le genre, les sexualités

$05 \mid 2018$

Raconter les sexualités depuis la marge

\section{Société de l'aveu, cis-tème de l'aveu : repenser le consentement à la lumière des images intimes de personnes trans* dans les medias}

Confessing Society, Confessing Cis-tem: Rethinking Consent Through Intimate Images of Trans* People in the Media

\section{Alexandre Baril}

Traducteur : Catriona LeBlanc

\section{(2) OpenEdition}

\section{Journals}

Édition électronique

URL : http://journals.openedition.org/glad/1260

DOI : $10.4000 /$ glad. 1260

ISSN : 2551-0819

Éditeur

Association GSL

Référence électronique

Alexandre Baril, « Société de l'aveu, cis-tème de l'aveu : repenser le consentement à la lumière des images intimes de personnes trans* dans les medias », GLAD! [En ligne], 05 | 2018, mis en ligne le 15 décembre 2018, consulté le 17 décembre 2020. URL : http://journals.openedition.org/glad/1260 ; DOl : https://doi.org/10.4000/glad. 1260

Ce document a été généré automatiquement le 17 décembre 2020.

\section{(i) $\odot$

La revue GLAD! est mise à disposition selon les termes de la Licence Creative Commons Attribution Pas d'Utilisation Commerciale - Pas de Modification 4.0 International. 


\title{
Société de l'aveu, cis-tème de
} l'aveu : repenser le consentement à la lumière des images intimes de personnes trans* dans les medias

\section{Confessing Society, Confessing Cis-tem: Rethinking Consent Through Intimate} Images of Trans* People in the Media

\author{
Alexandre Baril \\ Traduction : Catriona LeBlanc
}

\section{RÉFÉRENCE}

BARIL, Alexandre. 2017. «Confessing Society, Confessing Cis-tem: Rethinking Consent Through Intimate Images of Trans* People in the Media », in " Mapping Gendered Violence ", Frontiers: A Journal of Women Studies 39(2) 1-25.

\section{NOTE DE L'ÉDITEUR}

La version française de ce texte est une traduction de sa version originale anglaise parue dans : Frontiers : A Journal of Women Studies, 2018, vol $39 \mathrm{n}^{\circ} 2$ (p. 1-25), avec la permission de University of Nebraska Press (Copyright 2018).

\section{NOTE DE L'AUTEUR}

Remerciements : Certaines parties de ce texte ont été traduites de l'anglais par Catriona LeBlanc. Elle a aussi effectué le travail minutieux de lecture comparative entre 
les versions française et anglaise du texte ainsi que la révision complète de l'article. Je la remercie chaleureusement pour son travail.

Remerciements inclus dans la publication originale : Texte traduit du français par Catriona LeBlanc. Cette recherche a été généreusement soutenue par la bourse postdoctorale Izaak Walton Killam offerte par le Département de Science politique de l'Université Dalhousie. Des versions préliminaires de cet article ont été présentées en mai 2016 lors du congrès annuel de l'association Women's and Gender Studies/ Recherches féministes (WGSRF) et du $84^{\mathrm{e}}$ congrès de l'Association Francophone pour le Savoir (Acfas). J'aimerais remercier les participant-es de ces congrès pour leurs questions et commentaires. J'aimerais également remercier les directrices du numéro spécial de la revue Frontiers ainsi que les deux évaluatrices anonymes de cet article pour leurs utiles suggestions. J'aimerais offrir des remerciements chaleureux à l'assistante de recherche, Celeste Orr, qui a contribué rigoureusement à la recension des écrits pour cette recherche, de même que Jean-Sébastien Sauvé, Anne Dagenais Guertin et Catriona LeBlanc pour leur lecture attentive et leurs commentaires à propos de ce texte.

1 En tant que personne trans et chercheur universitaire spécialisé sur les enjeux trans*, j'ai reçu au cours des dix dernières années plus de cinq cents demandes de la part des journalistes et des médias, me demandant de participer à une grande variété de reportages, de films, de documentaires, d'émissions de variétés, de capsules télévisuelles, etc. Bien que ne pouvant être réduites à un tout homogène, ces demandes des médias étaient néanmoins majoritairement animées par une curiosité insatiable au sujet de ma transition et guidées par un désir de «montrer» ma transformation corporelle et de « raconter» le récit intime de ma transition au public. Cette «volonté de savoir " (Foucault 1976) des médias au sujet des personnes trans* se traduit souvent à travers des représentations médiatiques stéréotypées axées sur le récit autobiographique de la transition et des clichés visuels qui incluent des images intimes ${ }^{1}$. Comme plusieurs autres personnes trans*, j'ai participé, dans les premières années de ma transition, à des projets médiatiques impliquant l'immortalisation d'images intimes de ma transition (images nues), pour lesquelles il devient impossible par la suite de retirer le consentement, puisque le consentement à la diffusion d'images dans les médias, contrairement au consentement sexuel, représente un évènement unique et irrévocable. Malheureusement, je ne suis pas seul à faire face à la douloureuse réalité de l'étalage de ma vie intime dans l'espace public et de la diffusion publique d'images intimes de moi-même dans les médias contre mon gré. En janvier 2016, le juge David Stinson de la Cour suprême de l'Ontario a rendu un jugement sans précédent au Canada dans la cause Jane Doe c. N.D. ${ }^{2}$, un cas concernant la diffusion d'images photo/vidéographiques intimes. Dans cette affaire, un homme a été condamné à verser un montant de 141708 \$ en dommages-intérêts à son ex-conjointe après avoir diffusé, sans son consentement, une vidéo d'elle sur un site pornographique ainsi qu'à des connaissances. Le juge Stinson a évoqué à plusieurs reprises que ce cas pouvait "[...] de plusieurs manières [être] semblable à une agression sexuelle ${ }^{3}$ ». Suivant la tendance actuelle au Canada pour réformer les lois sur la porno-vengeance, la violence sexuelle et la cyberintimidation dans les médias sociaux, le jugement réfère aux modifications apportées au Code criminel du Canada en 2014 « [...] pour inclure une nouvelle infraction criminelle, soit la "publication non consensuelle d'une image intime" $[. . .]^{4} »$ passible de cinq années d'emprisonnement. En janvier 2016, le Manitoba 
est aussi devenu la première province à mettre en œuvre la Loi sur la protection des images intimes. Le durcissement des lois à l'égard de la diffusion d'images intimes en ligne et dans les médias n'est pas étranger aux cas tragiques d'Amanda Todd et de Rehtaeh Parsons, deux jeunes Canadiennes victimes de cyberintimidation qui se sont suicidées (Hasinoff 2015). Leurs histoires, dont la vidéo-témoignage de Todd visionnée près de vingt millions de fois (Todd 2012), ont non seulement fait le tour de l'actualité internationale, mais soulignent aussi la fréquence, les conséquences dévastatrices et les résultats parfois mortels de ces actes.

2 Si le jugement du juge Stinson et la Loi sur la protection des images intimes semblent fournir une prise pour des personnes qui, comme moi, souffrent de la diffusion de leur vie privée dans la sphère publique malgré leurs appels répétés à empêcher cette exposition, ces recours ne sont toutefois accessibles qu'aux victimes «idéales » dans des circonstances précises. En effet, ces jugements, nouvelles lois et amendements au Code criminel reposent sur la prémisse que la distribution de ces images intimes est dommageable parce qu'elle est non-consensuelle; l'intimité et la dignité sont violées lorsque des images destinées à une seule personne sont diffusées sur la place publique. Mais qu'en serait-il si un formulaire de consentement avait été signé ? Comment rétracter son consentement ou arrêter la diffusion d'images intimes dans de tels cas? Qu'adviendrait-il si le consentement avait été obtenu de manière coercitive, non pas selon les définitions couramment reconnues par la loi, comme la force physique ou l'alcool, mais par des formes de coercition plus diffuses et subtiles, comme les normes hétérosexistes et l'injonction à la monstration de son corps dans nos sociétés de l'image?

3 Malgré une redéfinition féministe du consentement sexuel axée sur son caractère renouvelable, affirmatif et rétractable, le consentement légal à la diffusion publique d'une image, que vous soyez en train de cuisiner ou de vous déshabiller devant la caméra, ne requiert qu'un accord unique et irrévocable. Vous ne pouvez jamais changer d'idée. La jurisprudence récente qui cite les conséquences négatives de la circulation d'images intimes et la compare à des formes d'agressions sexuelles ne s'applique pas à vous. Dans une logique néolibérale et individualiste qui blâme les victimes, signer un formulaire de consentement vous rend responsable. Toutefois, n'est-il pas problématique et déshumanisant de considérer le consentement à exposer sa nudité dans l'espace public au même titre qu'un consentement à montrer d'autres images de soi ou à acheter une voiture ? Le consentement à la diffusion publique d'images intimes devient éternel et ne peut être annulé, et ce, sans égard aux sentiments que pourrait éprouver la personne plus tard. Ma question est la suivante: peut-on redéfinir le consentement à la diffusion publique d'images intimes selon la compréhension féministe du consentement sexuel?

4 Cet article soutient qu'il est nécessaire de reconceptualiser le consentement à la diffusion d'images intimes dans les médias. Bien que cet argument s'applique à toute personne, dont les membres de divers groupes marginalisés, je m'attarderai ici aux personnes transgenres et transsexuelles (ci-dessous trans* avec un astérisque, pour signaler l'inclusion de l'ensemble des identités de genre variables). Que mon objectif soit clair: cette intervention ne prétend pas s'inscrire dans les champs des études juridiques ou médiatiques féministes, trop éloignés de mes champs de spécialisation en philosophie et en études féministes et de genre. Inspiré par des féministes comme Lise Gotell (2008; 2012), Melanie Randall (2010), Elizabeth Sheehy (2012) et Carrie 
Rentschler (2014), pour qui le droit n'est ni l'unique ni le meilleur outil pour combattre la culture du viol, je crois que le droit ne constitue qu'un outil parmi plusieurs pour lutter contre la «société de l'aveu» (Foucault 1976) et le cis-tème de l'aveu qui poussent les personnes trans* à se mettre à nu, figurativement et littéralement. Le mot "cis-tème " désigne un système créé par et pour les personnes cis. Le terme "cis " s'oppose à «trans» et, combiné aux mots «sexuel» et "genre» ("cissexuel», "cisgenre»), il désigne les personnes qui ne changent pas leur sexe/genre (Baril 2015a). Les termes « cissexisme » (Serano 2007), « cisnormativité » (Bauer et coll., 2009) et « cisgenrisme/cisgenrenormativité » (Baril 2015a ; 2015b) font référence au système oppressif qui discrimine les personnes trans* et au sein duquel les personnes cis constituent la norme. À partir de cette perspective, cet essai théorique offre des questionnements, des assemblages conceptuels et des réflexions critiques pour (re)penser la trans-formation du consentement que peuvent vivent certaines personnes trans* par rapport à la diffusion d'images intimes témoignant de leur parcours de transition. De ce point de vue, le consentement n'est pas un évènement unique, mais un processus, et ces réflexions pourront peut-être servir d'inspiration aux personnes travaillant à redéfinir la notion juridique du consentement ou dans le champ des études médiatiques féministes. J'espère surtout inviter les mouvements sociaux, les médias et le grand public à adopter des perspectives critiques sur le sens que pourrait prendre le consentement dans une société qui cultiverait une éthique médiatique soucieuse des groupes qui souffrent démesurément du sexisme, de l'hétéronormativité, du cisgenrisme, du racisme et du capacitisme.

5 À partir d'une approche interdisciplinaire, intersectionnelle, féministe et transactiviste, cet article mobilise les archives et les matériaux publiés en études féministes et trans* et adopte une méthodologie auto-ethnographique fondée sur ma propre expérience comme homme trans ayant "accepté » de me mettre à nu dans les médias, des images pour lesquelles mon consentement ne peut pas être retiré. Je présente deux arguments distincts, mais non exclusifs. Le premier soutient que, pour certaines personnes trans*, des conjonctures personnelles, communautaires, sociales et médiatiques particulières peuvent parfois créer une période d'hyper-visibilité qui peut biaiser leur consentement à la diffusion publique d'images intimes d'elles. Sans proposer une lecture hyper-structurelle pouvant nier l'agentivité des personnes trans*, je décris comment certains facteurs, dont l'insistance des médias à voir les " preuves " du «changement de sexe » et la sur-sexualisation généralisée des corps trans*, constituent des pressions qui, combinées à l'extrême vulnérabilité que peut entraîner une transition, créent des conditions propices pouvant altérer le jugement au regard de telles images. Je soutiens ainsi que les personnes trans* devraient être en mesure de retirer leur consentement à la diffusion publique de leurs images intimes sur la base d'un biais de consentement. Ce premier argument expose les facteurs structurels qui complexifient la notion néolibérale et individualiste du consentement. À partir de cet ancrage théorique, le second argument propose une redéfinition plus générale du consentement à la diffusion publique d'images intimes: que le consentement ait été donné dans des circonstances biaisées ou non, celui-ci devrait, comme c'est le cas du consentement sexuel, pouvoir être retiré en tout temps. S'il est possible de changer d'idée dans le cas d'activités intimes avec une seule personne, cela devrait aussi s'appliquer lorsqu'il est question de montrer son sexe, son intimité et sa nudité à un public potentiellement illimités. En m'inspirant des travaux féministes sur les agressions sexuelles et sur la notion de consentement, la seconde section déconstruit 
les conceptualisations néolibérales binaires (ex. les «bonnes» versus «mauvaises » victimes) qui réduisent les violences sexuelles à des problèmes individuels tout en occultant les facteurs structurels qui empêchent de concevoir les invasions médiatiques des intimités trans* comme des formes potentielles d'agressions à caractère sexuel lorsque les personnes trans* résistent, expriment leur inconfort ou demandent que de telles images ne soient pas diffusées publiquement. En conclusion, je lance une invitation à cultiver une éthique médiatique responsable envers les réalités des groupes marginalisés.

\section{Cis-tème de l'aveu : hyper-visibilité trans* et pressions à consentir}

En m'inspirant des travaux de Foucault sur l'aveu, j'argumente d'abord que nous vivons dans une «société de l'aveu » qui nous mène à croire que l'aveu public de nos désirs, de nos idées et de notre soi constitue une libération, ainsi que dans un «cis-tème de l'aveu » qui pousse les personnes trans* à se dénuder corps et âme devant un public avide de connaître tous les détails de leur transition. Foucault (1976: 79) explique l'importance de l'aveu dans un régime de pouvoir disciplinaire :

[L]'aveu est devenu, en Occident, une des techniques les plus hautement valorisées pour produire le vrai. Nous sommes devenus, depuis lors, une société singulièrement avouante. L'aveu a diffusé loin ses effets : dans la justice, dans la médecine, dans la pédagogie, dans les rapports familiaux, dans les relations amoureuses, dans l'ordre le plus quotidien, et dans les rites les plus solennels; on avoue ses crimes, on avoue ses péchés, on avoue ses pensées et ses désirs, on avoue son passé et ses rêves, on avoue son enfance ; on avoue ses maladies et ses misères ; on s'emploie avec la plus grande exactitude à dire ce qu'il y a de plus difficile à dire [...]. On avoue - ou on est forcé d'avouer. Quand il n'est pas spontané, ou imposé par quelque impératif intérieur, l'aveu est extorqué ; on le débusque dans l'âme ou on l'arrache au corps.

7 Cette injonction à l'aveu est d'autant plus individualisée dans une société où les médias sociaux permettent à tout le monde de tout partager. Depuis que les médias se sont intéressés à Christine Jorgensen il y a plus de cinquante ans (Meyerowitz 2002), leur intérêt pour les personnes trans* et les « changements de sexe » demeure constant. Que ce soit à la télévision ou dans les vidéos autoréalisées sur YouTube, les personnes trans* « avoue[nt] - ou [...] [sont] forcé[es] d'avouer » (Foucault 1976: 79) leur parcours de transition. Dans les cabinets des médecins, sur les plateaux de télévision, dans les études ethnographiques et dans leurs espaces privés, les personnes trans* sont appelées à avouer. J'insisterai sur quatre dimensions de ce cis-tème de l'aveu: personnelle, communautaire, sociale et médiatique. Je soutiens que ce cis-tème crée une «temporalité d'hyper-visibilité trans*6 " qui peut biaiser le consentement à la diffusion publique d'images intimes pour certaines personnes trans*.

\section{La dimension subjective du cis-tème de l'aveu : révéler un corps autrefois caché}

8 La première dimension du cis-tème de l'aveu concerne l'aspect subjectif et personnel de la transition. Les personnes trans* qui suivent un traitement hormonal vivent une seconde puberté physiologique, psychologique et affective (Cameron 1996; Green 2004 ; 
Baril 2016 ; 2017). Vivre une transition et découvrir un corps longtemps espéré peut créer une temporalité particulière (Halberstam 2005) ; cette période "d'adolescence " est marquée par l'exploration de ce corps et de cette nouvelle identité sociale (Hayward 2010) qu'il faut apprendre à révéler et à dissimuler selon le contexte. La satisfaction à l'égard de ce nouveau corps en développement est partie prenante d'un désir de le rendre visible pour la première fois. Alors que cet apprentissage du rapport au corps s'effectue chez les personnes cis lors de leur puberté, plusieurs personnes trans* doivent le réapprendre au moment de transitionner. Souvent, pendant de longues années, les personnes trans* nient et cachent leur identité et leur corps, comme c'était mon cas. Lorsque l'identité sociale et le corps commencent à se transformer, ils deviennent ainsi parfois l'objet d'un fort désir d'exposition (Cameron 1996 : 10-11). Ce désir d'avouer et de parler de notre transitude est encouragé par la société de l'aveu dont parle Foucault et est accentué par le cis-tème de l'aveu. Tout en appelant aux témoignages de nos identités trans* et même en nous obligeant à parler de notre transition à partir de la "volonté de savoir " (Foucault 1976) cisgenre, le cis-tème constitue aussi un dispositif de pouvoir dans lequel les identités trans* sont dévalorisées, délégitimées, dénigrées et discriminées, ce qui incite plusieurs personnes trans* à nier leur désir de transition. Simultanément, les violences, l'invisibilisation constante et les nombreuses difficultés personnelles, professionnelles, sociales, culturelles, politiques, juridiques et médicales vécues par les personnes trans* peuvent créer un besoin de se rendre visible et de parler des problèmes vécus ${ }^{7}$. Néanmoins, lorsque les personnes trans* sont invitées à parler de leurs expériences, l'intérêt du public se limite souvent aux aspects très personnels et principalement physiques de la transition ; les enjeux structurels sont négligés. Namaste (2012:479) écrit :

Les journalistes s'intéressaient surtout à la possibilité de me faire passer une entrevue formelle pour leur émission. Mais leurs questions se limitaient à mon histoire personnelle. Combien de temps avais-je vécu comme femme? Quand ai-je subi ma chirurgie? Étais-je contente des résultats? Comment ma famille a-t-elle réagi à ma transformation? J'ai expliqué aux journalistes et à l'équipe de réalisation que cela ne m'intéressait pas de raconter mon histoire personnelle. J'ai précisé, cependant, que j'étais disponible pour faire une entrevue à titre d'experte dans le domaine [...]. Pourtant, il n'y a peut-être pas lieu de s'étonner que les journalistes aient continué d'essayer de me convaincre d'accepter les termes d'une autobiographie. (traduction libre)

9 Si j'admire la force et le courage dont Namaste a fait preuve pour résister à cette invasion médiatique dans sa vie privée, il faut rappeler que des enjeux complexes liés à la race, la classe, le niveau d'éducation et l'âge, pour ne nommer que ces éléments, influencent la capacité des personnes trans* à résister à ces demandes insistantes de la part des médias. Étant donné qu'un parcours de transition est bouleversant et difficile, il n'est pas surprenant que certaines personnes trans* puissent avoir de la difficulté à dire non ou même ressentir un besoin de s'exprimer qui les pousse à répondre à ces questions. Comme l'explique Cameron (1996: 15), les expériences de la transitude et de la transition peuvent mener à un désir ambivalent de partager les détails intimes de nos vies, ce qui augmente notre vulnérabilité face à d'éventuelles exploitations :

Chaque fois que je dis à quelqu'un que je suis une personne transsexuelle, je vis une série d'émotions turbulentes. [...] [S]i l'accueil est positif, je commence à tout dévoiler avec un enthousiasme peu prévoyant, répondant à chaque question, ce qui en encourage toujours une autre. [...] En me dévoilant, j'ai consensuellement invité leur voyeurisme [...]. Leur attention et leur accueil stimulent mon désir de raconter [...]. [C]ependant, [cela] m'amène à des indiscrétions compulsives, et je dévoile 
facilement tout ce qui est personnel. [...] [Q]uand j'ai tout dit ou épuisé leur intérêt, je commence à me retirer un peu. Une ombre se pose sur moi, et je me rends compte que je ne me sens pas en sécurité. Je me sens nu. [...] Je commence à penser que je me suis exploité moi-même : j'ai honte de mon exhibitionnisme. (traduction libre)

10 Combinée à la fascination, la découverte et la volonté d'une personne trans* d'exposer une nouvelle corporéité et identité, cette injonction à parler de nos transitions d'un point de vue autobiographique et personnel et de révéler nos corps sexués/genrés incitent plusieurs personnes trans* à participer à ce cis-tème de l'aveu.

\section{La dimension communautaire du cis-tème de l'aveu : favoriser le changement social à travers la visibilité}

11 La deuxième dimension du cis-tème de l'aveu est liée à l'implication fréquente des personnes trans* dans des communautés qui encouragent la visibilité et la fierté. Beaucoup de personnes trans*, particulièrement dans les premières années d'une transition, se joignent à des groupes de soutien, cherchent l'aide des personnes trans* plus expérimentées et, graduellement, certaines d'entre elles prennent racine dans des communautés trans*, activistes ou non (Namaste 2000; ASTT(e)Q 2012). Comme les groupes gais, lesbiens, bisexuels et queers et d'autres groupes marginalisés, les communautés trans* ont démontré l'importance pour les personnes trans* de se rendre visibles dans la sphère publique ${ }^{8}$. Face à des sociétés hétéronormatives et cisgenrenormatives qui ont longtemps confiné les personnes marginalisées au silence, les groupes activistes militent avec raison en faveur d'une plus grande visibilité publique, une des revendications clés de plusieurs groupes (Cameron 1996). Toutefois, la visibilité n'est pas toujours souhaitée ni possible pour toutes les personnes trans*. Les travaux empiriques de Katrina Roen (2002) et d'autres déconstruisent le débat sur l'(in)visibilité et montrent que la visibilité d'une personne trans* varie selon le contexte, ses besoins, son sentiment de sécurité et d'autres facteurs. Malgré cette complexité, la visibilité représente toujours un enjeu central au sein des communautés trans*, au point de constituer parfois une injonction à la visibilité. Pour certaines personnes trans*, leur engagement auprès de communautés trans*, les encouragements d'être fières et visibles et les invitations à parler publiquement de leurs expériences d'oppression peuvent contribuer à l'hyper-visibilité. Cela étant dit, je ne dénonce aucunement ces invitations à la visibilité dans les communautés trans*, qui remplissent d'importantes fonctions sociales et politiques et qui ont des implications positives pour les personnes trans* qui sortent du silence. Tout comme le besoin de parler de ses expériences subjectives, le besoin de se rendre visible constitue une forme de résilience et de reprise de pouvoir face à la cisgenrenormativité. Mon objectif est de montrer que l'invisibilisation sociale cis-témique et l'absence de reconnaissance, parmi d'autres formes d'oppression, contribuent considérablement au désir de visibilité publique. Loren Cameron (1996: 10), qui a publié le premier livre dédié à rendre visibles des images d'hommes trans* et qui souligne l'importance de la visibilité trans* dans la sphère publique, parle de l'importance d'exposer son nouveau corps afin d'obtenir une reconnaissance sociale de son genre :

Je me suis photographié et j'ai envoyé des clichés amateurs à mes proches et à ma famille pour leur montrer à quel point j'étais content ; je voulais qu'ils s'habituent à l'idée que mon corps était différent. S'ils pouvaient voir ma nouvelle barbe et ma poitrine sans seins, peut-être qu'il serait plus facile pour eux d'accepter ma 
nouvelle identité. Tu sais, pour qu'ils arrêtent de m'appeler « elle». J'étais excité, aussi, tout comme je l'étais quand j'ai découvert ma sexualité à l'adolescence. Mais cette fois-ci, je refusais d'avoir honte. (traduction libre)

12 Combinée aux encouragements de leur communauté de pair-es, cette invisibilisation sociale peut provoquer chez les personnes trans* un sentiment d'urgence d'être visible en toutes circonstances et à tout moment, un besoin qui peut ou non changer au fil du temps. Par conséquent, le cis-tème de l'aveu ainsi que les résistances qui s'organisent pour le combattre peuvent favoriser une hyper-visibilité trans* qui prend souvent la forme de l'aveu public dans le cadre d'activités d'engagement communautaire.

\section{La dimension sociale du cis-tème de l'aveu : créer des subjectivités trans* dépossédées d'elles-mêmes}

Troisièmement, le cis-tème de l'aveu fonctionne aussi à travers un ensemble de conjonctures sociales qui mythifient et objectifient les "changements de sexe » (Cromwell 1999 ; Serano 2007). Ces conjonctures stimulent une hyper-visibilité trans* et façonnent sa représentation publique. Les personnes en transition sont constamment sollicitées à témoigner publiquement sur des enjeux comme les hormones, les chirurgies, la sexualité et les drames familiaux. Étant donné que les sociétés cisgenristes se sentent légitimes de les bombarder de questions, les personnes trans* se retrouvent souvent sur-sollicitées par leurs collègues de travail, leur famille, leurs connaissances ou les médias. Dès lors que l'identité trans* est révélée, elle devient souvent le seul point d'intérêt. Il devient difficile, dans ce contexte, de résister à l'aveu : si les conjonctures communautaires ou personnelles ne nous ont pas déjà poussé-es à tout avouer et nous rendre visibles, que cet acte soit «spontané, ou imposé par quelque impératif intérieur» (Foucault 1976: 79), les conjonctures sociales «forcent » l'aveu, pour reprendre la terminologie foucaldienne. Qu'il s'agisse d'institutions étatiques qui exigent le récit autobiographique pour changer de nom ou de mention de sexe, des employeurs qui requièrent des justifications pour « autoriser » l'utilisation des toilettes ou le port des uniformes, ou des familles qui exigent des explications sous peine d'exclusion, l'aveu des personnes trans* est « extorqué » (Foucault 1976 : 79). Et comme le souligne Namaste (2012: 480), pour les personnes trans*, l'injonction à l'aveu vise le corps et la sexualité plutôt que les problèmes structurels que nous vivons :

L'impératif autobiographique exige que les personnes transsexuelles racontent l'histoire de nos changements de sexe sur demande, que nous parlions de nos corps, de nos sexualités, de nos désirs, de nos organes génitaux et de notre douleur profonde selon les caprices d'une personne curieuse non transsexuelle. Il exige que nous racontions tout cela [...] sur demande. Et, par extension, il assure que nous n'aurons pas le temps, l'espace ou l'autorisation d'aborder les enjeux politiques et institutionnels sous-jacents qui rendent nos vies tellement difficiles: le contexte juridique du changement de nom ou les politiques administratives qui régissent l'assurance-maladie dans le cas de chirurgies de changement de sexe et d'autres services liés au transsexualisme. (traduction libre)

14 Foucault (1976: 79) observe que, pour plusieurs personnes marginalisées, comme c'est le cas des femmes, des personnes racisées, queers, intersexes et trans*, l'aveu peut aussi être « arraché » au corps. Par exemple, jusqu'en octobre 2015 au Québec, les personnes trans* devaient subir des chirurgies modifiant leurs capacités reproductives et leurs organes génitaux afin de pouvoir changer la mention de sexe sur le plan civil pour ensuite faire changer leurs papiers d'identité (Namaste 2000; ASTT(e)Q 2012 ; Baril 
2015b). Ces exigences, qui résultent en des stérilisations forcées, sont toujours en vigueur dans plusieurs pays et dans certaines provinces canadiennes [au moment de la rédaction de l'article, en 2016]. J'ai vécu, comme une majorité de personnes trans* canadiennes, une stérilisation forcée afin d'avoir accès à un état civil correspondant à mon genre d'auto-identification. Soulignons que je devais non seulement fournir le rapport opératoire du chirurgien, mais aussi le rapport d'un second médecin, ayant vérifié par un examen physique que l'opération avait été effectuée. La " vérité » du sexe et l'aveu d'une personne trans* sont ici arrachés au corps à coup de scalpels et de spéculums.

Pour certaines personnes trans*, ces aveux arrachés des corps et des âmes peuvent créer un sentiment de dépossession de soi semblable à celui que vivent parfois les personnes victimes d'agressions sexuelles (Randall 2010). Par ailleurs, une activiste intersexe me racontait comment, à 10 ans, alors qu'elle était dans le bureau d'un médecin pour un problème de peau sur le bas d'une fesse, elle a baissé sans pudeur son pantalon et sous-vêtement, avant même que le médecin ne lui demande. La réaction de surprise du médecin l'a à son tour surprise, réalisant que sa nudité était à la fois rapide et non nécessaire. Mais, à l'âge de 10 ans, c'était ce qu'elle avait appris en tant que personne intersexe : l'obligation de se mettre à nu pour les médecins (Preves 2002). Cette anecdote m'a incité à réfléchir sur le fait que l'on s'attend à ce que des personnes en transition exposent inutilement leur nudité. En effet, l'invasion fréquente et inappropriée des médecins sur nos corps à travers leurs examens, l'intérêt pour nos résultats chirurgicaux et les questions posées sur nos organes génitaux, nos orgasmes et d'autres sujets sensibles peuvent nous désensibiliser et créer un sentiment de dissociation de nos corps sexués. Cette obligation de se mettre à nu, au sens propre et figuré, se répercute sur la façon dont certaines personnes trans* présentent leur corps et leur nudité dans la sphère publique. Autrement dit, lorsqu'il faut répondre à des centaines de questions sur ses organes génitaux et se déshabiller si souvent chez le médecin, il devient plus facile de permettre aux médias d'accéder à son corps. La dimension sociopolitique du cis-tème de l'aveu peut ainsi déposséder les personnes trans* de leur corps, de leur nudité et de leur sens d'intimité et les propulser dans une économie de désinhibition et d'hyper-visibilité semblable à celle décrite par Cameron (1996).

\section{La dimension médiatique du cis-tème de l'aveu : « Montre-moi ton âme, montre-moi ton corps »}

Les personnes transsexuelles peuvent peut-être parler, mais seulement à condition qu'elles offrent leurs autobiographies personnelles et seulement lorsqu'elles répondent aux questions d'entrevue posées par une personne non transsexuelle.

(Namaste $2012: 478$, traduction libre)

16 Les médias contribuent aussi à l'hyper-visibilité trans* et constituent la quatrième composante du cis-tème de l'aveu. Bien que les médias s'intéressent de plus en plus aux enjeux trans*, l'intérêt public envers les personnes trans* date déjà d'une cinquantaine d'années (Meyerowitz 2002). La majorité des analyses portant sur le traitement médiatique des enjeux trans* critique la surexploitation de cette thématique en vue de 
se faire un capital culturel, sans égard au bien-être des communautés concernées ${ }^{9}$. Alors que l'étude des médias a démontré que l'exploitation sensationnaliste de certaines personnes est fréquente (Garland-Thomson 2007; Zoonen 2009; Richardson 2010; Sarkar 2014) dans des sociétés où les corps des femmes, des personnes queers, intersexes, trans*, racisées et d'autres groupes marginalisés sont objectifiés à des fins commerciales, les corps trans* deviennent ainsi une cible parfaite pour satisfaire la curiosité du public. Les journalistes, documentaristes, photographes et artistes sont avides de documenter ces transformations " extrêmes ", ces corps et ces sexualités. Ils veulent voir le corps et l'âme trans*; il faut montrer les «preuves » du "changement de sexe ». L'aspect sensationnaliste et la réduction des identités et des corps au statut de "freak» dans les médias ne se limitent pas aux identités trans*; comme le démontrent les spécialistes en études des médias, d'autres groupes, dont les personnes en situation de handicap et les personnes queers, sont ciblés par les «freak shows » (Gamson 1998 ; Garland-Thomson 2007 ; Richardson 2010). De plus, comme nous le rappellent souvent les spécialistes des médias et du cinéma, le genre documentaire est particulièrement bien adapté à ce type d'enquête, qui vise les détails intimes de la vie des gens (Spence et Navarro 2011; Ellis 2012). Bref, l'intérêt démesuré des médias envers les corps sexués et les organes génitaux des personnes trans* peut mener à une hyper-visibilité trans*. Étant donné les discriminations, les violences et les rejets souvent vécus pendant une transition, l'attention " positive » des médias peut avoir un certain attrait et amener certaines personnes à se soumettre à leurs demandes. Le problème n'est pas en soi l'intérêt des médias envers les personnes trans*, mais plutôt que nos récits et aveux doivent suivre un script très précis. Comme l'observe Namaste, se concentrer sur le corps sexué et sur la sexualité occulte les problématiques centrales qui touchent les communautés trans*

[...] dans chacun de ces exemples, la question de la privatisation des soins de santé pour les personnes transsexuelles n'est pas abordée. Cette absence souligne les limites importantes d'un cadre autobiographique. Viser exclusivement le quoi et le pourquoi de la transsexualité [...] empêche l'analyse critique des contextes institutionnels, économiques et politiques dans lesquels ont lieu les changements de sexe. (Namaste $2012: 480$, traduction libre)

Julia Serano décrit aussi comment la discrimination, les violences et d'autres difficultés sont évacuées dans les émissions et les documentaires sur les personnes trans* :

Plusieurs d'entre nous font face à de la discrimination en milieu de travail, à du harcèlement policier et à la menace constante de violence. Pourtant, les médias s'y intéressent très peu. Au lieu de cela, les émissions et les documentaires sur les personnes transsexuelles ont tendance à se concentrer presque exclusivement sur une dimension particulière de nos vies: nos transitions physiques. Ces émissions axées sur la transition semblent toujours suivre le même format, qui est composé d'échanges rigoureux sur toutes les interventions médicales qui y sont associées (hormones, chirurgies, électrolyse, etc.) et une abondance de photos avant-après apparemment incontournables. (Serano $2007: 53$, traduction libre)

18 Malgré le fait que mes différentes interventions au sein des médias aient toujours insisté sur les problématiques sociales, culturelles, politiques, économiques, juridiques, médicales que vivent les personnes trans* dans des sociétés cisgenristes, à plusieurs reprises, ce qui a été retenu de mon discours dans divers projets médiatiques auxquels j'ai participé a été axé sur l'aspect personnel et intime de ma transition, se conformant ainsi au script dominant décrit par Namaste et Serano, un script "façonné en vérité » (« crafted as truth »), pour reprendre l'expression de Spence et Navarro (2011). Les choix 
des profesionnel-les œuvrant dans les médias ont cherché à mettre l'accent sur le rapport à mon corps, me montrer nu et parler de ma bisexualité, alors que ces segments ne représentent qu'une petite portion des heures de vidéo tournées et des photos prises. Ces choix des médias confirment le concept en études médiatiques selon lequel les cinéastes créent des récits, même dans les documentaires (Ellis 2012), validant ainsi les critiques de plusieurs auteur-es trans* sur les représentations médiatiques des personnes trans*. Malgré la visibilité grandissante des personnes et des enjeux trans* dans les médias, la majorité des représentations demeurent conformes au script cisgenrenormatif dominant. Certes, toutes les représentations médiatiques n'empruntent pas cette voie et le script cis dominant n'a pas que des effets négatifs ; la réception de ces images et de ces témoignages n'est pas homogène et peut donner lieu à diverses interprétations. Ces représentations peuvent contribuer à sensibiliser le public, à briser les tabous et à aider d'autres personnes trans* (Cameron 1996; Green 2004 ; Shelley 2008 : 135). D'ailleurs, plusieurs personnes trans* inspirées par ma propre histoire et d'autres gens pour qui mes représentations dans les médias ont ouvert l'esprit ont communiqué directement avec moi pour témoigner de l'impact positif que ces représentations ont eu sur elles. Ce que je déplore, c'est le manque de diversité des représentations médiatiques des personnes trans* et le cis-tème de l'aveu qui cadre ces témoignages selon un seul script cis dominant.

\section{Hyper-visibilité trans*, consentement et médias}

Je soutiens que le cis-tème de l'aveu en général dans lequel se trouvent les personnes trans* et, plus précisément, ses quatre dimensions, soit personnelle, communautaire, sociale et médiatique, peuvent contribuer à un besoin d'hyper-visibilité trans*. Bien que je sois un homme éduqué et spécialisé sur les enjeux trans* ayant d'abord refusé de faire le récit autobiographique et de montrer ma transition dans les médias, j'ai moimême cru que je devais passer à l'aveu public de mon identité et permettre aux médias de « voir " ma transition. Notre " société de l'aveu » (Foucault 1976: 79), fondée sur un cisgenrisme et ses mécanismes connexes, m'a amené à accepter ce que je jugeais et juge encore aujourd'hui inacceptable: me déshabiller devant la caméra alors que je ne le voulais pas. Je désirais plutôt partager mon expertise sur les réalités trans*, sensibiliser le public aux difficultés que nous vivons et témoigner pour inspirer d'autres personnes trans*, mais le format médiatique exigeait que je révèle et parle de ma sexualité et de mes organes génitaux. Comme beaucoup de personnes trans* (Green $2004: 174$; Serano 2007 : 63-64 ; Shelley 2008 : 134-137), j'espérais que mon témoignage aiderait d'autres gens, mais en voyant mon propos construit selon des trames narratives prédéterminées (Spence et Navarro 2011) et soutenu par des images objectifiantes et morcellantes de mon corps, j'ai compris que j'avais, en faisant confiance aux personnes œuvrant dans les médias, commis une erreur.

Par ailleurs, les personnes trans* se retrouvent souvent dans une situation de vulnérabilité intensifiée en période de transition à cause des différentes problématiques et violences structurelles rencontrées (Cameron 1996; Green 2004 ; Baril 2015b ; 2017). La question que j'aimerais soulever ici nous ramène au propos central de cet article: étant donné la grande vulnérabilité lors d'une période de transition et les pressions à l'hyper-visibilité, comment une personne peut-elle faire des choix éclairés sur la diffusion d'images de son corps, de son intimité et de sa 
nudité ? Je soutiens que, combinée aux quatre dimensions du cis-tème de l'aveu, la période de vulnérabilité et d'hyper-visibilité temporaire vécue lors d'une transition peut amener certaines personnes trans* à prendre des décisions moins réfléchies quant à la diffusion publique d'images intimes d'elles-mêmes. J'argumente que ces conjonctures devraient, comme c'est le cas pour les drogues, la contrainte, l'influence d'une autorité ou encore les handicaps mentaux/émotionnels, compter parmi les motifs pouvant invalider un consentement si une personne trans*, au moment où elle retrouve une temporalité un peu plus "normale", regrette le fait d'avoir exposé publiquement son intimité, sa sexualité et sa nudité ${ }^{10}$. Je ne suggère pas que les personnes trans* sont inaptes à décider pour elles-mêmes dans d'autres sphères d'activités, ni qu'elles ne sont pas en mesure de prendre des décisions éclairées à propos de leur corps et de leur transition, ni qu'elles sont inaptes en général quant à la gestion de leurs apparitions publiques dans les médias. Mon argument est que certaines personnes trans* sont placées dans des conjonctures extraordinaires qui poussent à une hyper-visibilité de leur identité et de leur corps et qu'il s'agit d'un élément qui devrait être pris en considération comme facteur pouvant biaiser ou invalider le consentement. À l'instar de féministes travaillant dans le domaine des médias, notamment Amy Adele Hasinoff (2015), qui nous mettent en garde contre une vision trop déterministe de la participation des jeunes femmes au «sexting " qui nierait leur agentivité et leur autonomie à consentir, je ne crois pas que le cisgenrisme fait en sorte que les personnes trans* soient incapables de consentement autonome. S'il est important d'éviter la panique publique entourant le "sexting" et capital de rejeter la criminalisation comme solution unique aux problèmes systémiques (Hasinoff 2015), il faut tout autant éviter une "panique des médias» quant aux enjeux trans*. Néanmoins, je pense qu'il est nécessaire de proposer des conceptualisations plus robustes et plus éthiques du consentement concernant la diffusion d'images intimes dans les médias.

21 Contrairement au consentement sexuel, qui a fait l'objet d'une redéfinition vigoureuse dans plusieurs contextes législatifs, dont au Canada ${ }^{11}$, le consentement à la diffusion publique d'images intimes ne doit pas être constamment renouvelé et ne peut pas être retiré. Cela est en partie compréhensible ; si chaque personne qui accepte d'apparaître dans les médias pouvait retirer son consentement à tout moment, les équipes de réalisation ne voudraient peut-être pas investir l'argent et l'énergie nécessaires pour créer des productions pour lesquelles leurs droits d'auteur pourraient être retirés. Cela étant dit, je soutiens que le consentement à montrer l'image d'une personne en train de jardiner ou de cuisiner n'est pas la même chose que le consentement à la diffusion publique d'images dans lesquelles cette personne est nue ou exhibe d'une manière ou d'une autre son intimité, sa vie privée ou sa sexualité. Or, aucune distinction n'est faite entre ces deux types de consentement: le formulaire de consentement, éternel et irrévocable lorsque signé, s'applique de la même façon que la personne soit nue ou en train de cuisiner. Toutefois, qu'arrive-t-il lorsque des personnes qui, au nom de leur liberté d'expression journalistique, du droit de savoir du public ou de l'art, utilisent les images et les témoignages de personnes trans* d'une manière qui les rend mal à l'aise, les vulnérabilise et les expose à de potentiels dangers, comme le harcèlement public, la discrimination, la perte d'emploi, et plus encore? Quelles sont les conséquences négatives sur les plans social, psychologique, affectif et relationnel de ces mises à nu publiques non seulement pour les personnes trans*, mais également pour leurs familles? Je crois que la diffusion d'images intimes dans les médias doit être redéfinie. Néanmoins, des changements aussi profonds prennent du temps et, en attendant une 
telle révolution dans la manière de concevoir le consentement eu égard aux images intimes, des réformes, bien que limitées, pourraient aider à contrer cette exploitation des identités et des corps trans*. À défaut d'une redéfinition complète du consentement à la diffusion publique d'images intimes, nos systèmes juridiques pourraient néanmoins revoir les conditions sous lesquelles il est difficile de consentir librement.

\section{Repenser le consentement : images intimes de personnes trans* dans les médias}

Les travaux féministes sur la question du consentement portent principalement sur les femmes (les hommes s'en trouvent souvent exclus ou vus comme toujours consentants ou violant le consentement [Hequembourg 2015]) et les discussions entourant le consentement se situent dans un cadre hétéronormatif et cisgenrenormatif. Sous cet angle, mon identité d'homme trans et bisexuel rend souvent inintelligibles mes expériences d'agressions sexuelles, qu'il s'agisse de celles vécues dans mon enfance en tant que personne auparavant identifiée femme ou, plus tard dans ma vie, dans mon identité actuelle d'homme trans. S'il est capital de s'intéresser au consentement à partir de la perspective des femmes hétérosexuelles puisqu'une majorité écrasante de la violence sexuelle est perpétrée par des hommes sur des femmes, il ne faut pas oublier la violence sexuelle que subissent les populations LGBQ et intersexes (Preves 2002), non binaires, genderqueers, ou trans ${ }^{* 12}$ (Bauer et Scheim 2015). Dans sa recension des écrits portant sur des études quantitatives répertoriant les formes de violence exercées envers les personnes trans*, Stotzer (2009:3) rapporte qu'environ la moitié des personnes trans* a subi des agressions sexuelles : « la constatation la plus fréquente dans l'ensemble des sondages et des évaluations des besoins est qu'environ $50 \%$ des personnes transgenres affirment avoir vécu des activités sexuelles non désirées " (traduction libre). Étant donné que la violence sexuelle peut prendre plusieurs formes, incluant les comportements du personnel médical à l'égard des corps sexués/genrés des personnes intersexes et trans* (Meyerowitz 2002 ; Bauer et coll. 2009 ; Preves 2002), la violence sexuelle ne devrait pas être réduite aux activités sexuelles forcées. Autrement dit, comme féministes, nous devons élargir nos définitions de ce qui constitue des violences qui portent atteinte au sexe, au genre et à la sexualité. Afin de repenser le consentement et la question des violences sexuelles, je propose de déconstruire trois binarités conceptuelles : celle qui oppose les activités sexuelles aux représentations sexuelles, celle qui oppose la sexualité consensuelle à la sexualité nonconsensuelle, et celle qui oppose les «bonnes » victimes aux «mauvaises » victimes. Ces processus de déconstruction, utilisés avec beaucoup de succès par les féministes, n'ont pas encore été appliqués aux enjeux trans* ni aux formes de violences sexuelles vécues par les personnes trans*.

\section{Déconstruire les binarités : activités versus représentations sexuelles}

Bien que certaines auteures, dont MacKinnon (1987), aient, dans les années 1980, interrogé les liens entre les activités et les représentations sexuelles - une période caractérisée par d'importantes tensions entre féministes et connue en anglais comme 
les «sex wars» ("guerres du sexe ») -, les analyses féministes pro-sexes sur ces liens demeurent rares. La perspective radicale de MacKinnon au regard de la violence sexuelle, qui met sur un pied d'égalité les dimensions symboliques et physiques de la violence sexuelle, ne fait pas l'unanimité au sein des communautés féministes. Alors que certains travaux (ex. Sarkar 2014) soutiennent la pertinence de placer ces formes de violence sur un même continuum, d'autres soulignent les limites de cette perspective (Rentschler 2014 ; Hasinoff 2015). Les travaux sur le consentement sexuel mettent l'accent sur l'acte sexuel plutôt que sur la représentation de la sexualité ou sur les images intimes ou à caractère sexue ${ }^{13}$. Mais peut-on vraiment dissocier l'acte sexuel de la représentation de la sexualité et de la nudité ? À l'ère numérique, on voit émerger des préoccupations pour la porno-vengeance et les images intimes et à caractère sexuel, particulièrement en ce qui concerne les jeunes qui se retrouvent dans des situations déplorables, comme les deux jeunes femmes présentées en introduction (Canada, Ministère de la Justice 2013). Bien que je ne propose pas de rendre les violences représentationnelles ou symboliques indifférenciables des violences physiques, car une telle approche occulterait les spécificités de chacun de ces types de violence, je remets toutefois en question notre adhésion à des cadres limités qui nous empêchent de percevoir les conséquences psychologiques et parfois physiques pouvant résulter de violences symboliques. Malgré un intérêt grandissant pour la violence symbolique, les quelques discussions émergentes sur ce sujet ne concernent toutefois que le contrôle de la diffusion publique "non-consensuelle» de ces images et de la violence qui les accompagne, comme la vengeance et l'intimidation, qui existe indépendamment $\mathrm{du}$ matériel photo/vidéographique en soi. Étant donné qu'elle présente des images intimes et à caractère sexuel auxquelles des personnes ont " consenti ", la "violence ordinaire » de ces images intimes circulant dans les médias, de même que les conséquences dévastatrices que peuvent avoir ces dernières, demeurent impensées dans le cas des personnes qui changent d'avis ou qui aimeraient retirer leur consentement.

Les arguments présentés plus haut nous invitent à repenser la notion de consentement à la diffusion publique d'images intimes. Partant du postulat que le consentement d'une personne trans* à exposer son corps et sa nudité ne devrait pas être compris comme un consentement à acheter un téléphone ou à apparaître dans une émission de jardinage, je pose la question suivante : le consentement à la diffusion publique d'images intimes ne s'apparente-t-il pas davantage au consentement sexuel ? Étant donné que certains contextes juridiques nous permettent désormais de retirer notre consentement lorsqu'il est question de révéler notre intimité à une seule personne, ne serait-il pas logique de pouvoir retirer notre consentement à l'exposition de notre intimité à un public potentiellement illimité ? Pour certaines personnes, les conséquences d'une telle exposition peuvent être aussi graves que celles d'une agression sexuelle. Une fois situé dans le contexte d'autres conceptualisations, plus éthiques, du consentement, comme le consentement médical ou les formulaires utilisés dans les recherches universitaires (Kahn et coll. 1998) qui permettent aux personnes participantes de retirer à tout moment leur consentement en ce qui concerne les renseignements d'entrevue et d'autres données, souvent moins intimes que des images de nudité ou des témoignages sur leur sexe, genre et sexualité, le fait que les médias ne requièrent qu'un seul consentement pour la diffusion publique d'images (intimes) se distingue comme une forme d'exceptionnalisme. Les travaux féministes ${ }^{14}$ ont déjà montré que le consentement sexuel doit être affirmatif, constamment renouvelé et devrait pouvoir 
être retiré à tout moment, et la décision du juge Stinson dans le cas de pornovengeance insiste à plusieurs reprises sur les conséquences dévastatrices à long terme de la diffusion d'images intimes dans les médias :

Ces dernières années, les technologies ont permis aux personnes aux comportements prédateurs et d'intimidation de victimiser les autres en diffusant sans consentement leurs photos nues ou vidéos intimes. Nous comprenons maintenant les effets dévastateurs qui peuvent découler de ces actes, allant du suicide des adolescentes victimes de ces actes, aux conséquences pouvant mettre fin à la carrière lorsque les victimes sont des personnes établies. [...] $]^{15}$. (traduction libre)

Sur la base de ces conséquences négatives, le juge Stinson a accordé des dommagesintérêts sans précédent en droit canadien. Toutefois, ce jugement, comme les réformes proposées aux lois canadiennes sur la diffusion d'images intimes, se fondent sur le nonconsentement, et non pas sur les images en elles-mêmes, leurs implications sur la vie de la personne ou les traumas psychologiques qui peuvent en découler, malgré l'existence d'un formulaire de consentement signé dans des circonstances très précises. Comme personne ayant vécu des agressions sexuelles dans mon enfance, je peux témoigner en toute sincérité que j'ai vécu la médiatisation d'images intimes sur ma transition et l'accessibilité publique de ces images par mes collègues, mes ami-es, ma famille et mes centaines d'étudiant-es, comme une violation, dont les impacts psychologiques s'apparentent fortement à la violence sexuelle : conséquences négatives sur l'estime de soi et la vie sexuelle, dépression, et plus encore (Jozkowski et Peterson 2013). Le fait que j'aurais "consenti» à la diffusion de ces images intimes, selon une notion hétérosexiste, cisgenriste et néolibérale très étroite de la loi en ce qui concerne le consentement aux images dans les médias, ne diminue en rien son impact désastreux dans ma vie. Malheureusement, mon cas n'est pas exceptionnel. Un nombre grandissant de personnes trans* et les personnes qui s'allient à leur cause, dont des personnages publics comme Janet Mock et John Oliver, s'élèvent pour dénoncer l'objectification, la sexualisation et la fétichisation des corps trans*16. C'est la raison pour laquelle nous devons problématiser la conceptualisation binaire entre les actes qui portent atteinte à notre intimité sexuelle et les images qui peuvent aussi la violer.

\section{Déconstruire les binarités : sexualité consensuelle versus non- consensuelle}

Il existe un problème fondamental sous-tendant le jugement du juge Stinson et d'autres décisions juridiques concernant la diffusion publique d'images intimes: le consentement au sens légal est conceptualisé en vase clos, en évacuant le rôle des enjeux systémiques et structurels et des relations de pouvoir. Tandis qu'une personne n'ayant pas consenti reçoit une protection juridique, une personne ayant donné son consentement n'est pas comprise comme une victime et ne mérite donc aucune protection juridique. Or, comme le rappelle Beres (2007: 99), les travaux féministes soulignent l'importance d'une lecture plus nuancée, complète, contextualisée et politisée du consentement :

Le consentement devient quelque chose de plus large que tout simplement dire « oui » à la sexualité avec une personne particulière, dans un lieu particulier et à un moment particulier. Il devient une négociation d'attentes sociales, une façon d'exprimer son identité sociale ou d'intégrer un certain monde social. Il crée des 
espaces pour la sexualité qui ne sont ni consensuels ni criminels ou violents, bien qu'ils puissent être problématiques sur le plan social. (traduction libre)

À l'instar des féministes ayant démontré que la notion néolibérale et individualiste du consentement déployée dans les appareillages juridiques fait porter le blâme aux victimes (Gotell 2008; 2012; Randall 2010 ; Sheehy 2012), j'argumente que la notion actuelle du consentement à la diffusion publique d'images intimes repose sur une vision néolibérale et individualiste de l'autonomie. Sous cet angle, le consentement est accordé ou refusé par un sujet neutre, autonome et libre de pressions sociales ou culturelles, une perspective qui ne tient pas compte de l'influence des facteurs structurels. Sans réduire la subjectivité et l'agentivité individuelles aux forces structurelles, je crois que, pour les personnes trans*, le cis-tème de l'aveu et ses dimensions personnelle, communautaire, sociale et médiatique peuvent constituer une forme de coercition sociale (Gavey 1989; Randall 2010), différente de la coercition interpersonnelle. Si ces deux formes de coercition peuvent avoir des effets désastreux, seule la seconde est actuellement prise en considération par la loi. Gavey écrit (1989 : 468) :

Il est donc important de se pencher sur tout l'éventail de la coercition sexuelle qui se produit au sein de ces relations. Cela comprend les phénomènes peu traités de la coercition sociale (ex. participer à des actes sexuels uniquement pour éviter, par exemple, l'apparence de "frigidité » ou d'être démodé-e) et de la coercition interpersonnelle [...]. Ces formes plus normatives de coercition comprennent les situations où la femme semble consentir à participer à une interaction, malgré le fait de ne pas vouloir. Certaines de ces formes de coercition, particulièrement la coercition sociale, sont rarement discutées dans la littérature sur la victimisation sexuelle. (traduction libre)

28 Ni le cis-tème de l'aveu, ni la coercition sociale ne sont pris en compte dans les prises de décision des personnes trans* quant à leurs témoignages publics ou le partage d'images intimes. À une époque où une image peut faire le tour du monde en quelques heures et hanter une personne pour toujours, il semblerait important de réfléchir à ces formes de pressions lors de l'évaluation de la capacité d'une personne trans* de prendre des décisions éclairées quant à l'exposition publique de son intimité. Pour ce faire, il est capital de déconstruire la conceptualisation binaire du consentement qui oppose le consentement au non-consentement à la diffusion publique d'images intimes, surtout puisque, comme le montrent mon histoire et celle de plusieurs autres personnes trans* relatant leurs expériences des médias (Cromwell 1999; Green 2004 ; Namaste 2012), la réalité est plus complexe.

Les analyses féministes des violences sexuelles veulent aller au-delà d'une lecture individualiste et néolibérale de ces agressions sexuelles et de l'opposition entre le consentement et le non-consentement; elles adoptent une perspective axée sur les inégalités systémiques vécues entre hommes et femmes dans nos sociétés hétérosexistes pour comprendre et montrer l'influence de ces structures sur le consentement et les prises de décision des femmes ${ }^{17}$. Burkett et Hamilton (2012: 820) observent que la sexualité est divisée en catégories binaires en fonction de normes dominantes selon lesquelles les activités sexuelles sont soit consensuelles, soit nonconsensuelles. Mais la réalité est beaucoup plus compliquée. Burkett et Hamilton (2012 : 822-828) montrent que plusieurs éléments rendent difficile la possibilité de dire non à des rapports sexuels pour certaines femmes, notamment la confiance, les pressions indirectes verbales ou physiques, la culpabilité, les normes internalisées sur la sexualité, l'évitement des conflits, la peur de décevoir l'autre, le désir d'être reconnue, 
etc. Ces éléments, qui interfèrent dans la décision de "consentir » aux activités sexuelles potentiellement non désirées, s'appliquent également aux images des personnes trans* dans les médias.

Nous voulons, comme personnes trans*, faire confiance aux professionnel-les œuvrant dans les médias. Des arguments sont présentés pour nous convaincre de faire des choses que nous ne voulons pas faire et nous nous y soumettons parfois de peur de les décevoir ou pour éviter les conflits. Parce que ces personnes professionnelles s'intéressent à notre histoire, nous sentons l'obligation de leur donner quelque chose en retour. De plus, les relations de pouvoir entre les personnes cis et trans*, comme c'est le cas entre hommes et femmes, ne sont pas égalitaires et il peut être difficile pour plusieurs personnes trans* de négocier le consentement dans cette relation hiérarchique. S'il est difficile pour une femme de dire non aux avances sexuelles d'un homme après avoir accepté de souper avec lui et de se rendre à son appartement, imaginez la difficulté de personnes trans* à dire non à des personnes des médias avec qui elles auraient collaboré à plusieurs reprises ou même sur plusieurs années, comme c'est le cas des personnes trans* qui participent à des documentaires sur leur transition. Imaginez la culpabilité et la peur de refuser la vision de cinéastes et réalisateurs-trices cisgenres qui sont convaincues qu'il faut du nu pour toucher le public. Les «bons" sujets trans*, ceux qui suivent le script cisgenrenormatif des médias, se plient à cette injonction de se mettre à nu, corps et âme. À la lumière de ces faits, je soutiens que le "consentement" de certaines personnes trans* à la diffusion publique d'images intimes est compris comme valide à partir d'une lecture individualiste et néolibérale, qui privatise et décontextualise (Burkett et Hamilton 2012: 830) les expériences de violences, notamment sexuelles, que vivent les personnes trans* au quotidien et dans les médias. Qu'elles regrettent ou non le partage de leur transition sur la place publique, les personnes trans* sont tenues entièrement responsables de leurs «mauvais » choix dits personnels. Je pense qu'il est temps de déconstruire cette binarité du consentement à la diffusion publique d'images intimes des personnes trans* (et d'autres personnes) selon laquelle une personne est soit consentante, soit non consentante, puisque la réalité entourant ces « choix » est beaucoup plus complexe.

\section{Déconstruire les binarités : « bonnes » versus « mauvaises » victimes}

Plusieurs féministes ont dénoncé les prémisses néolibérales qui sous-tendent nos conceptualisations et nos jugements de ce que sont les agressions sexuelles. Gotell $(2008 ; 2012)$ soutient que le contexte néolibéral individualise les enjeux systémiques et structuraux et réduit la culture du viol et la violence sexuelle à des problèmes isolés, ce qui exige des femmes qu'elles adoptent des pratiques de sécurité personnelle et des stratégies individuelles de gestion des risques. Malgré la réforme des lois sur les agressions sexuelles, les définitions actuelles du consentement font en sorte que les femmes sont responsables d'éviter de se faire violer et de se mettre « en danger ». Cela mène à la pratique courante qui consiste à blâmer la victime ; une femme est violée lorsqu'elle "échoue» à se protéger adéquatement ou lorsqu'elle prend trop de " risques ». Il s'agit également de l'interprétation dominante quant aux personnes qui consomment des drogues et de l'alcool, qui exercent le travail du sexe et qui sont 
identifiées comme des femmes autochtones ou racisées (Pietsch 2009; Randall 2010). Gotell écrit (2008 : 889) :

Le discours juridique du consentement affirmatif crée une séparation entre les évènements individuels et les relations de pouvoir qui construisent les vulnérabilités. Les dernières sont réduites au silence et les limites imposées sur les actions survenant dans les situations de marginalisation sociale sont effacées. De cette manière, les agressions sexuelles sont individualisées, dépolitisées et reconstruites comme un échec de la responsabilisation, tandis que les relations de pouvoir qui définissent les violences sexuelles sont obscurcies. La vulnérabilité est reconstruite comme échec de sa responsabilité [...]. (traduction libre)

C'est la raison pour laquelle plusieurs campagnes menées contre les violences sexuelles se sont éloignées de l'approche «non, c'est non ». Ce type de slogan responsabilise la victime, dont le refus aurait pu être plus clair, et néglige de problématiser la responsabilité de la personne qui l'agresse (Kitzinger et Frith 1999 ; Bussell 2008 ; Gotell 2008 ; Burkett et Hamilton 2012 ; Beres 2014). Malgré les réformes légales au Canada, le «mythe de la victime idéale » (Randall 2010) persiste au sein des mentalités, des médias et $\mathrm{du}$ traitement que réservent les cours de justice aux personnes agressées sexuellement. Ce mythe perpétue la distinction entre les «bonnes » et les « mauvaises » victimes d'agressions sexuelles (Gotell 2008; 2012; Randall 2010): alors que les premières sont légitimées, crues et protégées, les secondes sont délégitimées, blâmées pour leurs comportements "à risque » et ne reçoivent pas de protection (Gotell 2008 ; 2012 ; Pietsch 2009 ; Randall 2010).

Il n'est pas surprenant que cette même logique néolibérale soit à l'œuvre dans les cas impliquant les personnes trans*, dont certaines sont des femmes racisées, des travailleuses du sexe, séropositives ou consommatrices de drogues (Garafolo et coll. 2006, Clements-Nolle et coll. 2006 ; ASTT(e)Q 2012). Si les personnes trans* acceptent de participer à des documentaires, de parler ouvertement de leur "nouveau sexe » et de leur sexualité ou d'enlever leurs vêtements, elles sont étiquetées comme ayant des attitudes trop risquées et taxées de «mauvaises" victimes parce qu'elles auraient échoué à se prémunir du risque d'instrumentalisation et de sexualisation de leur corps par les médias. Bref, elles sont, comme les femmes agressées sexuellement qui ne correspondent pas à l'image des victimes « idéales », responsables de ce qui leur arrive. Selon cette logique néolibérale, les «bonnes» victimes doivent résister vigoureusement à la violence sexuelle (Randall 2010: 422). Les personnes trans* devraient donc, pour être considérées comme de «bonnes » et de « vraies » victimes, se montrer agressives envers les médias et leurs demandes, parce que simplement dire non, exprimer son malaise ou montrer des signes détresse ou de résistance (comme je l'ai souvent fait) ne constitue pas des preuves suffisamment claires qu'elles ne consentent pas à exposer leur nudité.

Inspiré par des travaux féministes qui tentent de contextualiser et de repolitiser la notion du consentement, je pense que la notion de consentement à la diffusion publique d'images intimes des personnes trans* (et d'autres personnes) devrait être repensée non pas comme un évènement, mais comme un processus (Humphreys 2004 ; Beres 2007 ; Beres 2014). Les recherches sur le consentement sexuel montrent que les hommes tendent à voir le consentement comme un évènement singulier, tandis que les femmes le voient davantage comme un processus. Sous cet angle, la conceptualisation $\mathrm{du}$ consentement à la diffusion publique d'images intimes relève d'une vision hétérosexiste et masculine dominante du consentement qui l'appréhende comme un 
évènement singulier et qui peut être tenu pour acquis sans égard aux autres signes de malaise qui pourraient survenir par la suite. Une réflexion critique sur l'éthique du consentement qui prend en considération les différents rapports de pouvoir hétérosexiste, raciste, cisgenriste, capacitiste, etc. ne peut que mener à une redéfinition de ce qui constitue, pour des groupes marginalisés, le consentement à la diffusion publique d'images de leurs corps, de leur sexualité et de leur intimité.

\title{
Cultiver une éthique des médias sensible aux communautés marginalisées
}

\author{
Une des obligations morales et éthiques des \\ journalistes critiques n'est-elle pas d'aborder \\ précisément les politiques sociales et les \\ pratiques institutionnelles qui condamnent les \\ personnes transsexuelles à la pauvreté abjecte et \\ à un profond isolement social ? \\ (Namaste $2012: 481$, traduction libre)
}

Comme Viviane Namaste, je pense que les journalistes, les cinéastes et les autres personnes travaillant dans les médias sur des enjeux sociaux et qui travaillent auprès de communautés marginalisées doivent non seulement adopter un point de vue critique sur les contenus présentés au public, mais également s'assurer que leurs produits ne marginalisent et n'exposent pas davantage ces groupes à la violence. Dans les cas précis de l'objectification et de la sexualisation des corps et des identités des personnes trans* dans les médias, il existe un besoin urgent de réfléchir collectivement aux conséquences négatives et à long terme que peut avoir l'immortalisation vidéographique et photographique dans notre ère numérique. Ce texte constitue une invitation à ouvrir un dialogue avec les personnes œuvrant au sein de divers médias pour les encourager à cultiver des approches éthiques complexes sur le consentement des groupes marginalisés, dont les personnes trans*, au regard de la diffusion publique de leurs images intimes. Qu'arrive-t-il lorsque le consentement n'est plus pensé comme étant accordé ou non, est compris comme un processus complexe plutôt qu'un évènement unique et est conceptualisé comme s'inscrivant dans des relations sociales au lieu d'être défini à partir d'une conception néolibérale du sujet et de son agentivité ? Malheureusement, plusieurs personnes travaillant dans les médias profitent, voire exploitent, la période d'hyper-visibilité trans* produite dans notre cis-tème de l'aveu afin de proposer des nouvelles, des films, des documentaires ou des produits artistiques sensationnalistes. Plutôt que d'exploiter cette hyper-visibilité et la vulnérabilité des personnes trans* en les immortalisant en photo ou en images vidéographiques, une éthique des médias plus responsable interrogerait les conditions d'émergence et les conséquences du désir d'hyper-visibilité trans*. Bref, des médias socialement responsables devraient exposer cette "volonté de savoir " (Foucault 1976) du cis-tème de l'aveu et l'exploitation de l'hyper-vibilité trans* plutôt que de l'exploiter et de l'utiliser pour se faire un capital culturel et financier. Il faudrait également réfléchir aux moyens de sensibiliser nos communautés marginalisées quant aux enjeux cruciaux liés aux témoignages publics sur nos identités, nos vies privées et nos sexualités et créer des outils qui nous permettraient de prendre des décisions plus éclairées quant à notre participation ou non à une variété de projets et d'activités médiatiques. Parce que 
le consentement n'est jamais l'affaire d'une seule personne, mais d'une collectivité, il est temps d'inviter davantage de personnes à prendre part à cette importante discussion.

\section{BIBLIOGRAPHIE}

ACTION SANTÉ TRAVESTI(E)S ET TRANSSEXUEL(LE)S DU QUÉBEC (ASTT(e)Q). 2012. « Taking Charge. A Handbook for Health Care and Social Service Providers with Trans People », consulté le 23 janvier 2016. URL : http://www.catie.ca/sites/default/files/ TakingChargeHandbookupdated.pdf.

BARIL, Alexandre. 2017. « Temporalité trans : identité de genre, temps transitoire et éthique médiatique » Enfances, familles, générations : Revue internationale 25, URL : http:// journals.openedition.org/efg/1359.

BARIL, Alexandre. 2016. « “Doctor, Am I an Anglophone Trapped in a Francophone Body?” An Intersectional Analysis of Trans-Crip-t Time in Ableist, Cisnormative, Anglonormative societies » Journal of Literary \& Cultural Disability Studies 10 (2) : 155-172.

BARIL Alexandre. 2015a. « Needing to Acquire a Physical Impairment/Disability: (Re)Thinking the Connections Between Trans and Disability Studies Through Transability » Hypatia 30 (1) :

$30-48$.

BARIL, Alexandre. 2015b. « Transness as Debility: Rethinking Intersections between Trans and Disabled Embodiments » Feminist Review $111:$ 59-74.

BAUER, Greta R., et SCHEIM, Ayden I. 2015. Transgender People in Ontario, Canada: Statistics from the Trans PULSE Project to Inform Human Rights Policy. London : University of Western Ontario.

BAUER, Greta R., et coll. 2009. « “I Don't Think This Is Theoretical; This Is Our Lives”: How Erasure Impacts Health Care for Transgender People » The Journal of the Association of Nurses in AIDS Care 20 (5) : 348-361.

BERES, Melanie Ann. 2007. « "Spontaneous” Sexual Consent: An Analysis of Sexual Consent Literature » Feminism \& Psychology 17 (1) : 93-108.

BERES, Melanie Ann. 2014. «Rethinking the Concept of Consent for Anti-Sexual Violence Activism and Education » Feminism and Psychology 24 (3) : 373-389.

BURKETT, Melissa, et Karine HAMILTON. 2012. « Postfeminist Sexual Agency: Young Women's Negotiations of Sexual Consent » Sexualities 15 (7) : 815-833.

BUSSEL, Rachel Kramer. 2008. «Beyond Yes or No: Consent as Sexual Process », in Yes Means Yes! Visions of Female Sexual Power and a World Without Rape, FRIEDMAN Jaclyn and Jessica VALENTI (ed.). Berkeley, CA : Seal Press, 43-52.

CAMERON, Loren. 1996. Body Alchemy: Transsexual Portraits. San Francisco : Cleis Press.

Canada, Department of Justice, Coordinating Committee of Senior Officials (CCSO) Cybercrime Working Group (CWG). 2013. Cyberbullying and the Non-consensual Distribution of Intimate Images, 
consulté le 23 janvier 2016. URL : http://www.justice.gc.ca/eng/rp-pr/other-autre/cndii-cdncii/ p1.html.

CLEMENTS-NOLLE, Kristen, et coll. 2006. « Attempted Suicide Among Transgender Persons: The Influence of Gender-Based Discrimination and Victimization » Journal of Homosexuality 51 (3) : 53 69.

CRAIG, Elaine. 2012. Troubling Sex: Towards a Legal Theory of Sexual Integrity. Vancouver : University of British Columbia Press.

CROMWELL, Jason. 1999. Transmen and FTMs: Identities, Bodies, Genders, and Sexualities. Urbana/ Chicago : University of Illinois Press.

CURRAH, Paisley (dir). 2006. Transgender Rights. Minneapolis : University of Minnesota Press.

Doe 464533 v N.D., 2016 ONSC 541. CanLII.

ELLIS, John. 2012. Documentary: Witness and Self-Revelation. New York : Routledge.

ESPINEIRA, Karine. 2008. La transidentité. De l'espace médiatique à l'espace public. Paris :

L'Harmattan.

ESPINEIRA, Karine, et Maud-Yeuse THOMAS. 2014. « Les trans comme parias. Le traitement médiatique de la sexualité des personnes trans en France » Genre, sexualité \& société 11 : 1-15.

FOUCAULT, Michel. 1976. Histoire de la sexualité : la volonté de savoir. Paris : Gallimard.

GAMSON, Joshua. 1998. Freaks Talk Back: Tabloid Talk Shows and Sexual Nonconformity. Chicago : Chicago University Press.

GARLAND THOMSON, Rosemarie. 2007. Extraordinary Bodies. Figuring Physical Disability in American Culture and Litterature. New York : Columbia University Press.

GAROFALO Robert, et coll. 2006. « Overlooked, Misunderstood and At-Risk: Exploring the Lives and HIV Risk of Ethnic Minority Male-to-Female Transgender Youth » Journal of Adolescent Health $38: 230-236$.

GAVEY, Nicola. 1989. « Feminist Poststructuralism and Discourse Analysis » Psychology of Women Quarterly 13 : 459-475.

GOTELL, Lise. 2008. « Rethinking Affirmative Consent in Canadian Sexual Assault Law: Neoliberal Sexual Subjects and Risky Women » Akron Law Review 41 (4) : 865-898.

GOTELL, Lise. 2012. « Third-Wave Anti-rape Activism on Neoliberal Terrain: The Garneau Sisterhood ", in Sexual Assault in Canada: Law, Legal Practice and Women's Activism, SHEEHY Elizabeth A. (dir). Ottawa : University of Ottawa Press, 243-265.

GREEN, Jamison. 2004. Becoming a Visible Man. Nashville : Vanderbilt University Press.

HALBERSTAM, Judith/Jack. 2005. In a Queer Time and Place. Transgender Bodies, Subcultural Lives, Sexual Cultures. New York : New York University Press.

HASINOFF, Amy Adele. 2015. Sexting Panic: Rethinking Criminalization, Privacy, and Consent. Champaign : University of Illinois Press.

HAYWARD Eva. 2010. « Spider City Sex » Women \& Performance: A Journal of Feminist Theory 20 : 225-251.

HEQUEMBOURG, Amy L., et coll. 2015. « Sexual Assault Risk Among Gay and Bisexual Men » Journal of Sex Research 52 (3) : 282-295. 
HUMPHREYS, Terry P. 2004. «Understanding Sexual Consent: An Empirical Investigation of the Normative Script for Young Heterosexual Adults ", in Making Sense of Sexual Consent, COWLING, Mark (dir). Aldershot : Ashgate, 207-225.

JOZKOWSKI, Kristen N., et Zoe D. PETERSON. 2013. «College Students and Sexual Consent: Unique Insights » The Journal of Sex Research 50 (6) : 517-523.

KAHN, Jeffrey, Anna C. MASTROIANNI et Jeremy SUGARMAN (dir). 1998. Beyond Consent: Seeking Justice in Research. Oxford : Oxford University Press.

KITZINGER, Celia, et Hannah FRITH. 1999. «Just Say No? The Use of Conversation Analysis in Developing a Feminist Perspective on Sexual Refusal » Discourse \& Society 10 : 293-316.

LANGENDERFER-MAGRUDER, Lisa, et coll. 2016. « Experiences of Intimate Partner Violence and Subsequent Police Reproting Among Lesbian, Gay, Bisexual, Transgender, and Queer Adults in Colorado: Comparing Rates of Cisgender and Transgender Victimization » Journal of Interpersonal Violence 31 (5) : 855-871.

LOMBARDI, Emilia, et coll. 2001. « Gender Violence: Transgender Experiences with Violence and Discrimination » Journal of Homosexuality 42 (1) : 89-101.

MACKINNON, Catharine. 1987. Feminism Unmodified. Discourses on Life and Law. Cambridge : Harvard University Press.

MEYEROWITZ, Joanne. 2002. How Sex Changed: A History of Transsexuality in the United States. Cambridge : Harvard University Press.

NAMASTE, Viviane K. 2000. Invisible Lives: The Erasure of Transsexual and Transgendered People. Chicago : The University of Chicago Press.

NAMASTE, Viviane. 2012. « Beyond Image Content: Examining Transsexuals’ Access to the Media ", in Queerly Canadian: An Introductory Reader in Sexuality Studies, FITZGERALD, Maureen and Scott RAYTER (dir). Toronto : Canadian Scholars' Press, 477-488.

PIETSCH, Nicole. 2009. « 'I'm Not That Kind of Girl:' White Femininity, the Other, and the Legal/ Social Sanctioning of Sexual Violence Against Racialized Women » Canadian Woman Studies/Les Cahiers de la Femme 28 (1) : 136-140.

PREVES, Sharon E. 2002. « Sexing the Intersexed: An Analysis of Sociocultural Responses to Intersexuality » Signs 27 (2) : 523-536.

Protecting Canadians from Online Crime Act, Statutes of Canada 2014, c.31, consulté le 23 janvier 2016. URL : http://laws-lois.justice.gc.ca/eng/annualstatutes/2014_31/

RANDALL, Melanie. 2010. « Sexual Assault Law, Credibility, and 'Ideal Victims': Consent, Resistance, and Victim Blaming » Canadian Journal of Women and the Law 22 (2) : 397-433.

RICHARDSON, Niall. 2010. Transgressive Bodies: Representations in Film and Popular Culture. Farnham : Ashgate.

RENTSCHLER, Carrie. 2014. « Rape Culture and the Feminist Politics of Social Media » Girlhood Studies 7 (1) : 65-82.

ROEN, Katrina. 2002. « 'Either/Or' and 'Both/Neither': Discursive Tensions in Transgender Politics » Signs 27 (2) : 501-522.

RYAN, Joelle Ruby. 2009. « Reel Gender: Examining the Politics of Trans Images in Film and Media ». PhD. Bowling Green : Bowling Green State University. 
SARKAR, Sumita. 2014. « Media and Women image: A Feminist Discourse » Journal of Media and Communication Studies 6 (3) : 48-58.

SERANO, Julia. 2007. Whipping Girl. A Transsexual Woman on Sexism and the Scapegoating of Femininity. Berkeley : Seal Press.

SHEEHY, Elizabeth A. (dir). 2012. Sexual Assault in Canada: Law, Legal Practice and Women's Activism. Ottawa : University of Ottawa Press.

SHELLEY, Christopher A. 2008. Transpeople: Repudiation, Trauma, Healing. Toronto : University of Toronto Press.

SPENCE, Louise et Vinicius NAVARRO. 2011. Crafting Truth: Documentary Form and Meaning. New Brunswick : Rutgers University Press.

STOTZER, Rebecca L. 2009. «Violence Against Transgender People: A Review of United States Data » Aggression and Violent Behavior 14 (3) : 170-179.

TODD, Amanda. 2012. « My Story: Struggling, Bullying, Suicide, Self Harm », Vidéo YouTube, 8:54 min, 7 septembre, consulté le 23 janvier 2016. URL : https://www.youtube.com/watch? $\mathrm{v}=\mathrm{vOHXGNX-E7E.}$

ZOONEN, Liesbet van. 2009 [1994]. Feminist Media Studies. London : SAGE Publications.

\section{NOTES}

1. Selon la Loi sur la protection des Canadiens contre la cybercriminalité, une "image intime" s'entend d'un enregistrement visuel - photographique, filmé, vidéo ou autre - d'une personne, réalisé par tout moyen, où celle-ci : a) y figure nue, exposant ses seins, ses organes génitaux ou sa région anale ou se livrant à une activité sexuelle explicite ; $b$ ) se trouvait, lors de la réalisation de cet enregistrement, dans des circonstances pour lesquelles il existe une attente raisonnable de protection en matière de vie privée; $c$ ) a toujours cette attente raisonnable de protection en matière de vie privée à l'égard de l'enregistrement au moment de la perpétration de l'infraction. " Mon usage de l'expression « image intime " suit la condition (a) et cherche à problématiser les conditions (b) et (c).

2. Doe 464533 v N.D., 2016 ONSC 541 (CanLII).

3. Traduction libre. Doe 464533 v N.D., 2016 ONSC 541, CanLII, [52].

4. Traduction libre. Doe 464533 v N.D., 2016 ONSC 541, CanLII, [18].

5. Cette réflexion critique sur le consentement à la diffusion publique d'images intimes ou de nudité est particulièrement pertinente dans le contexte de la présence toujours croissante des médias sociaux (Rentschler 2014 ; Hasinoff 2015). Il est important de rappeler que, dans la plupart des contextes nationaux, les lois sur le consentement à la diffusion publique d'images ont été établies avant l'existence généralisée d'Internet et des médias sociaux. Je soutiens que les nouvelles réalités technologiques et sociales exigent que nous repensions ces lois.

6. Pour une discussion de la temporalité trans*, voir Baril $(2016 ; 2017)$.

7. Voir Namaste 2000 ; Lombardi et coll. 2001 ; Roen 2002 ; Currah 2006 ; Serano 2007 ; Espineira 2008 ; Bauer et coll. 2009 ; Stotzer 2009.

8. Voir Cromwell 1999 ; Green 2004 ; Serano 2007 ; Espineira 2008.

9. Voir Halberstam 2005; Serano 2007; Espineira 2008; Shelley 2008: 132-140; Ryan 2009; Namaste 2012 ; Espineira et Thomas 2014.

10. Plusieurs personnes trans*, en raison du sentiment de dysphorie et des violences systémiques qu'elles subissent, vivent d'importants problèmes en santé mentale (Shelley 2008 ; Bauer et coll. 2009 ; Baril 2015b). Les études empiriques (Bauer et Scheim 2015) montrent que plus de 50\% des 
personnes trans* rapportent des états dépressifs, ce qui peut biaiser la capacité de consentir de façon éclairée.

11. Pour un historique de la notion du consentement affirmatif dans le droit canadien, voir la synthèse de Gotell (2008). Pour des définitions et des recensions des écrits à propos du consentement, voir Beres $(2007 ; 2014)$.

12. Voir Lombardi et coll. 2001; Clements-Nolle et coll. 2006; Garofalo et coll. 2006 ; Stotzer 2009 ; Langenderfer-Magruder et coll. 2016.

13. Voir Humphreys 2004 ; Bussell 2008 ; Pietsch 2009 ; Randall 2010 ; Burkett et Hamilton 2012 ; Sheehy 2012 ; Jozkowski et Peterson 2013 ; Beres 2014.

14. Voir Beres 2007 ; 2014 ; Gotell 2008 ; 2012 ; Randall 2010 ; Burkett et Hamilton 2012 ; Craig 2012 ; Sheehy 2012.

15. Doe 464533 v N.D., 2016 ONSC 541, CanLII, [16], [17].

16. Voir Oliver, John. « Last Week Tonight with John Oliver: Transgender Rights. » Vidéo YouTube. 16:46. 28 juin 2015. https://www.youtube.com/watch?v=hmoAX9f6MOc ; Mock, Janet. « Activist Janet Mock Flips the Script on Reporter: Asks Her to Prove Her Womanhood. » Vidéo YouTube. 3:07. 6 mai 2014. https://www.youtube.com/watch?v=ISsdSvJhniQ

17. Voir Kitzinger et Frith 1999 ; Humphreys 2004 ; Randall 2010 ; Jozkowski et Peterson 2013.

\section{RÉSUMÉS}

Malgré une redéfinition féministe du consentement sexuel axée sur son caractère renouvelable, affirmatif et rétractable, le consentement légal à la diffusion publique de tout type d'image de soi ne requiert qu'un accord unique et irrévocable. Je soutiens qu'il est problématique de considérer le consentement à la diffusion publique de sa nudité au même titre qu'un consentement à montrer d'autres images de soi. Peut-on redéfinir le consentement à la diffusion publique d'images intimes selon la compréhension féministe du consentement sexuel ? En m'inspirant des travaux de Foucault sur l'aveu, j'argumente que nous vivons dans une «société de l'aveu » qui nous mène à croire que l'aveu public de notre soi le plus profond constitue une libération, ainsi que dans un «cis-tème de l'aveu » qui pousse les personnes trans* à se dénuder corps et âme pour satisfaire la curiosité du public. À partir d'une approche féministe et transactiviste et adoptant une méthodologie auto-ethnographique fondée sur ma propre expérience comme homme trans ayant «accepté » des requêtes des médias de me mettre à nu et pour lesquels mon consentement ne peut pas être retiré, je présente deux arguments. Premièrement, pour certaines personnes trans*, certaines dimensions du cis-tème de l'aveu, dont les pressions sociales et médiatiques, devraient être prises en compte comme éléments pouvant biaiser le consentement. Deuxièmement, j'invite à repenser le consentement à la diffusion publique d'images intimes : que le consentement soit accordé dans des circonstances biaisées ou pas, il devrait être possible de le retirer, comme dans le cas du consentement sexuel. S'il est possible de changer d'idée dans le cas d'activités intimes avec une seule personne, cela devrait aussi s'appliquer lorsqu'il est question de montrer sa nudité au grand public.

Despite feminist redefinitions of sexual consent that highlight its renewable, affirmative, retractable nature, legal consent to the public distribution of any type of image requires only a single, irrevocable instance of consent. I argue that it is problematic to consider consent to publicly distribute intimate nude images in the same way as consent to sharing other images. 
What if consent to the public distribution of intimate images were redefined according to feminist understandings of sexual consent? Using the example of trans* people and inspired by Foucault's work on confessing, I argue that we live in a "confessing society" that leads us to believe that disclosing our deepest selves is liberating and a transphobic/cisgenderist "confessing cis-tem" that, together, push trans* people to show their naked bodies/souls to satisfy public curiosity. Adopting a feminist, transactivist approach and an auto-ethographic methodology based on my experience as a trans man who reluctantly undressed for media projects on my transition for which my consent cannot be retracted, I present two arguments. First, for some trans* people, certain aspects of the confessing cis-tem, including social and media pressures, should be considered factors that can bias consent. Second, I call for a redefinition of consent to the distribution of intimate images: whether consent is given in biased circumstances or not, it should be possible to withdraw it, as with sexual consent. If people can reconsider being intimate with just one other person, the same should be true of exposing their nudity to an audience.

\section{INDEX}

Thèmes : Recherches

Keywords : transgender, media, cisgenderism, transphobia, consent, sexual violence, visibility

Mots-clés : transgenre, médias, cisgenrisme, transphobie, consentement, violence sexuelle, visibilité

\section{AUTEURS}

\section{ALEXANDRE BARIL}

Alexandre Baril, Ph.D. en études des femmes, est professeur adjoint à l'École de service social de l'Université d'Ottawa et spécialiste de la diversité, incluant la diversité sexuelle et de genre, corporelle (handicaps et santé) et linguistique. La formation interdisciplinaire d'Alexandre Baril combine dix années en philosophie/éthique, un doctorat en études des femmes, ainsi que deux stages postdoctoraux en études féministes, des genres et des sexualités à Wesleyan University (Bourse postdoctorale du Conseil de recherches en sciences humaines du Canada/CRSH) et en science politique à Dalhousie University (Bourse postdoctorale Izaak Walton Killam). Il a publié plusieurs articles dans des revues telles que Hypatia : Journal of Feminist Philosophy; Feminist Review; TSQ : Transgender Studies Quarterly; Atlantis: Critical Studies in Gender, Culture \& Social Justice; Frontiers: A Journal of Women Studies; Annual Review of Critical Psychology; Medicine Anthropology Theory; Journal of Literary \& Cultural Disability Studies; Canadian Journal of Disability Studies; Disability \& Society; Recherches féministes; Enfances, familles, générations : Revue internationale; et Recherches sociologiques \& anthropologiques. Ses recherches intersectionnelles se situent à la croisée des études de genre, queer, trans, du handicap et de la sociologie du corps, de la santé et des mouvements sociaux. Ses travaux récents se penchent sur les discours tenus sur le suicide et le suicide assisté au sein des mouvements et des études anti-oppression. 